\title{
Short GSM mobile phone exposure does not alter human auditory
} brainstem response

\author{
Gábor Stefanics*1,3, Lóránd Kellényi1 ${ }^{1}$ Ferenc Molnár², Györgyi Kubinyi², \\ György Thuróczy² and István Hernádi ${ }^{1}$
}

Address: ${ }^{1}$ Department of Experimental Zoology and Neurobiology, University of Pécs, Hungary, ${ }^{2}$ Department of Non-ionizing Radiation, National "Frédéric Joliot-Curie" Research Institute for Radiobiology and Radiohygiene, Budapest, Hungary and 3institute for Psychology, Hungarian Academy of Sciences, Budapest, Hungary

Email: Gábor Stefanics* - gstefan@cogpsyphy.hu; Lóránd Kellényi - kellenyi@gamma.ttk.pte.hu; Ferenc Molnár - molnar@hp.osski.hu; Györgyi Kubinyi - bakos@hp.osski.hu; György Thuróczy - thuroczy@hp.osski.hu; István Hernádi - hernadi@ttk.pte.hu

* Corresponding author

Published: 12 November 2007

BMC Public Health 2007, 7:325 doi:10.1 I86/147/-2458-7-325
Received: 3 May 2007

Accepted: 12 November 2007

This article is available from: http://www.biomedcentral.com/I47I-2458/7/325

(C) 2007 Stefanics et al; licensee BioMed Central Ltd.

This is an Open Access article distributed under the terms of the Creative Commons Attribution License (http://creativecommons.org/licenses/by/2.0), which permits unrestricted use, distribution, and reproduction in any medium, provided the original work is properly cited.

\begin{abstract}
Background: There are about I.6 billion GSM cellular phones in use throughout the world today. Numerous papers have reported various biological effects in humans exposed to electromagnetic fields emitted by mobile phones. The aim of the present study was to advance our understanding of potential adverse effects of the GSM mobile phones on the human hearing system.
\end{abstract}

Methods: Auditory Brainstem Response (ABR) was recorded with three non-polarizing $\mathrm{Ag}-\mathrm{AgCl}$ scalp electrodes in thirty young and healthy volunteers (age 18-26 years) with normal hearing. ABR data were collected before, and immediately after a 10 minute exposure to $900 \mathrm{MHz}$ pulsed electromagnetic field (EMF) emitted by a commercial Nokia 6310 mobile phone. Fifteen subjects were exposed to genuine EMF and fifteen to sham EMF in a double blind and counterbalanced order. Possible effects of irradiation was analyzed by comparing the latency of ABR waves I, III and $\checkmark$ before and after genuine/sham EMF exposure.

Results: Paired sample t-test was conducted for statistical analysis. Results revealed no significant differences in the latency of $A B R$ waves I, III and $\mathrm{V}$ before and after 10 minutes of genuine/sham EMF exposure.

Conclusion: The present results suggest that, in our experimental conditions, a single 10 minute exposure of $900 \mathrm{MHz}$ EMF emitted by a commercial mobile phone does not produce measurable immediate effects in the latency of auditory brainstem waves I, III and V. 


\section{Background}

There are about 1.6 billion GSM mobile phones (MPs) in use throughout the world today. Due to the close proximity of the antenna of the mobile handset to the user's ear and head, the brain is inevitably exposed to EMFs with a relatively high specific absorption ratio (SAR). Results of experimental radiofrequency (RF) dosimetry indicate that approximately $40-55 \%$ of the mobile phone's RF output power energy is absorbed in the users head [1]. Due to the high number of MP users and the relative high SAR close to the ear, it is important to resolve whether or not EMF exposure by MPs can adversely affect the human hearing system.

Numerous studies have investigated the electrophysiological effects of EMF exposure in humans. A significant delay in the latency of the fifth wave $(\mathrm{V})$ of the ABR after $15 \mathrm{~min}$ of exposure to EMF emitted by a GSM MP was recorded [2] and the authors suggested that the observed delay might lead to a temporary 15-18 dB hearing deficiency above $2 \mathrm{kHz}$ in the normal hearing frequency range. On the other hand, a different study found no effects on ABR (I, III and V waves) after a 30 minute MP irradiation [3]. Studying the influence of MP EMFs on outer hair cell functions [4] revealed, that a single $10 \mathrm{~min}$ MP exposure did not induce any changes in the generation of distortion product otoacoustic emissions (DPOAE) in humans. Investigation of hearing threshold levels by pure tone audiometry and transient evoked otoacoustic emissions before and immediately after 10 min of genuine or sham exposure of MP EMF yielded no significant effect caused by the irradiation [5]. These results suggest that a single 10 minute exposure to EMF emitted by a MP has no immediate effects on hearing threshold levels. Investigation of the effects of EMF on later auditory event-related brain potentials also yielded controversial results. No significant effects of 30 min of MP exposure were found on middle latency responses [3]. The authors concluded that $30 \mathrm{~min}$ of MP irradiation had no short-term adverse effects on human auditory system. However, in an auditory oddball task, a significant decrease in the amplitude and latency of N100 component of the auditory evoked response to nontarget stimuli and an increase in the latency of the P300 to target stimuli were found as a result of one hour MP EMF exposure [6]. In this study, reaction times were significantly slower during EMF exposure, and the authors suggested that MP exposure might affect neuronal activity and alter human cognitive performance. However, a more recent study from the same group [7] investigated the possible effects of 30 min MP EMF on auditory and visual evoked responses in 120 normal subjects. In this followup study, previous positive findings were not replicated and the authors concluded that there was no evidence for any effects of acute MP EMF exposure on event-related brain potentials and reaction time in humans. In a recent study [8], somatosensory evoked potentials were recorded in 12 normal subjects before and after exposure to 30 minutes of MP EMF. The authors found that somatosensory cortex was not affected by exposure to EMF. Facilitating effects EMF emitted by MPs on human cognitive functions have been reported by several authors. A decrease in reaction time was observed in a choice reaction time task [9], in simple reaction time and vigilance tasks [10] and in a memory task during high memory load [11]. However, subsequent studies could not replicate these findings $[12,13]$. EMF exposure was reported to have significant effects on human brain oscillatory activity in the $8-10 \mathrm{~Hz}$ frequency band during a memory task [14], but memory performance was found similar during sham and genuine EMF exposure. Effects of MP EMF on preattentive processing and working memory were studied by event-related brain potentials during a working memory test [15]. The authors observed a significant effect of EMF exposure on the P50 component, whereas in their study memory performance was also not affected by EMF exposure.

Considering the possible biological effects of MPs used widely in daily communication as a high-priority environmental health issue, the European Commission (5th Framework Program for Research and Technological Development) launched the GUARD project: "Potential Adverse Effects of GSM Cellular Phones on Hearing" with the aim of addressing the potential effects of GSM MP exposure on the hearing system of laboratory animals and humans.

\section{Methods \\ Subjects}

The present experiments were carried out on 30 healthy volunteers (aged $24 \pm 5$ years, 15 women) with no clinical evidence of hearing disorders. The protocol of the study was approved by the Ethics Committee of the University of Pécs. All subjects gave their written informed consent after the nature of the experiment had been fully explained.

\section{Stimuli}

We delivered three types of acoustic stimuli to the subjects: condensation, rarefaction and alternating $100 \mu \mathrm{s}$ click sound stimuli. With this protocol we also aimed to test for possible different effects of EMF exposure on brainstem response to the above types of acoustic stimuli. We delivered 2048 clicks for each stimulus type twice to ascertain their reproducibility. The stimulus rate and intensity were set to $27 \mathrm{~Hz}$ and $80 \mathrm{~dB}$ SPL, respectively. Click stimuli were generated by a loudspeaker embedded in a $34 \mathrm{~cm}$ long sound tube causing an acoustic delay of 1 ms to separate the loudspeakers electromagnetic artifacts from brainstem responses. The acoustic delay tube had a 
sound damping textile lining on the inside surface attenuating acoustic reflection noises.

\section{Exposure setup and Specific Absorption Rate (SAR)}

During auditory brainstem response (ABR) recording and exposure subjects lay supine in a dimly lit, sound attenuated room on an electrically shielded bed with eyes closed. They were instructed to avoid unnecessary movements. Exposure was administered by means of a standard Nokia 6310 MP via external software control at a constant $2 \mathrm{~W}$ peak power for $10 \mathrm{~min}$. The MP was mounted on a plastic headset in normal use position. In order to evaluate and control the levels of EMF exposure, the Specific Absorption Rate (SAR, $\mathrm{W} / \mathrm{kg}$ ) was previously assessed for the inner ear region in a brain tissue equivalent liquid phantom device. Details of the SAR measurement procedure were described elsewhere [4]. Maximum peak SAR at a distance of $3 \mathrm{~cm}$ from the surface of the phantom, corresponding approximately to the position of the cochlea, was $0.41 \mathrm{~W} / \mathrm{kg}$ at $900 \mathrm{MHz}$ frequency.

\section{Audiometry}

The hearing status of participants for each ear was measured for both air $(125 \mathrm{~Hz}$ to $10 \mathrm{kHz})$ and bone $(250 \mathrm{~Hz}$ to $2 \mathrm{kHz}$ ) conducted sound stimuli. A clinical audiometer (Medicor ATK-5-N20-10-84) was used to obtain standard audiograms for all subjects. A high quality headphone (Telephonics Corporation P/N OC017) was used for auditory stimulus delivery. Hearing threshold levels (HTL) in both ears of 30 recruited subjects were no worse than 30 $\mathrm{dB}$ at the standard audiometric frequencies.

\section{$A B R$ recording conditions}

Subjects were randomly assigned to one of the following groups: EMF group (fifteen subjects exposed to genuine EMF irradiation, eight women), Control group (fifteen subjects exposed to sham EMF irradiation, seven women). According to our experimental protocol, the administration of genuine or sham exposure was double blind. Our study was carried out in close agreement with the protocol of the European Commission 5th Framework project "GUARD: potential adverse effects of GSM cellular phones on hearing [16]."

\section{$A B R$ recording and data analysis}

The auditory brainstem response (ABR) was recorded with three non-polarizing $\mathrm{Ag}-\mathrm{AgCl}$ electrodes. Electrode impedances were kept below $5 \mathrm{kOhms}$ measured at 15 $\mathrm{Hz}$. All electrodes were filled with standard EEG paste (TEN20, Weaver and Co., Aurora, CO). Resting DC electrode potentials were measured by an impedance meter. Differential potentials of the electrode pairs were set to less than $1 \mathrm{mV}$.

The active electrode was placed on the right mastoid and the reference electrode was placed over the vertex $(\mathrm{Cz}$ of the international 10-20 system). The ground electrode was placed on the forehead over the nasion and was connected to the active ground of the amplifier. The amplifier was set to a gain of $10 \mathrm{k}$. The lower and upper cut-off frequencies were set at $100 \mathrm{~Hz}$ and $3000 \mathrm{~Hz}$, respectively.

Continuous EEG signal was recorded with a sampling rate of $20 \mathrm{kHz}$ at 12 bit resolution (CED 1401, Cambridge Electronic Device Ltd, Cambridge, UK) and stored on a hard disk. Data processing and analysis was performed off-line with a custom-built MATLAB software routine written by one of the authors (GS) on a personal computer.

For each stimulus, an epoch of $15 \mathrm{~ms}$ duration including a $3 \mathrm{~ms}$ pre-stimulus period was extracted from the continuous EEG data. Epochs with a potential change below 0.1 $\mu \mathrm{V}$ or above $100 \mu \mathrm{V}$ were rejected from further analysis. Due to excessive muscle artifacts, three data sets were excluded from further analysis.

Latencies of wave I, III and V were measured for each stimulus type and subject. We studied the effects of stimulus condition on these values obtained before and after EMF exposure with paired Student's t tests using the Statistica software package (StatSoft).

\section{Results}

Tables 1, 2, 3 show the results of the statistical analysis and the mean latencies $( \pm$ standard deviation) of ABR wave I, III and V before and after MP EMF exposure for the

Table I: Mean ABR peak I latencies before and after genuine or sham RF exposure

\begin{tabular}{|c|c|c|c|c|c|c|}
\hline Condition & Stimulus type & $\mathbf{n}$ & Before RF (ms) & After RF (ms) & $\mathbf{t}$ & $\mathbf{P}$ \\
\hline \multirow[t]{3}{*}{ Genuine EMF } & Rarefaction & 13 & $1.67 \pm 0.14$ & $1.66 \pm 0.15$ & 0.34 & 0.737 \\
\hline & Condensation & 13 & $1.69 \pm 0.18$ & $1.65 \pm 0.19$ & 1.18 & 0.260 \\
\hline & Alternating & 13 & $1.79 \pm 0.09$ & $1.78 \pm 0.12$ & 1.06 & 0.310 \\
\hline \multirow[t]{3}{*}{ Sham EMF } & Rarefaction & 15 & $1.61 \pm 0.21$ & $1.63 \pm 0.19$ & -0.50 & 0.624 \\
\hline & Condensation & 14 & $1.66 \pm 0.09$ & $1.68 \pm 0.14$ & -0.24 & 0.807 \\
\hline & Alternating & 14 & $1.74 \pm 0.10$ & $1.76 \pm 0.11$ & -1.23 & 0.239 \\
\hline
\end{tabular}

Mean ABR peak I latencies ( \pm standard deviation) before and after genuine or sham 10 min $900 \mathrm{MHz}$ RF exposure generated by a commercial MP and results of the statistical analysis. 
Table 2: Mean ABR peak III latencies before and after genuine or sham RF exposure

\begin{tabular}{|c|c|c|c|c|c|c|}
\hline Condition & Stimulus type & $\mathbf{n}$ & Before RF (ms) & After RF (ms) & $\mathbf{t}$ & $\mathbf{p}$ \\
\hline \multirow[t]{3}{*}{ Genuine EMF } & Rarefaction & 13 & $4.08 \pm 0.19$ & $4.11 \pm 0.18$ & -0.52 & 0.610 \\
\hline & Condensation & 13 & $4.04 \pm 0.10$ & $4.05 \pm 0.15$ & -0.22 & 0.827 \\
\hline & Alternating & 13 & $4.13 \pm 0.12$ & $4.15 \pm 0.13$ & -0.75 & 0.468 \\
\hline \multirow{3}{*}{ Sham EMF } & Rarefaction & 15 & $4.03 \pm 0.21$ & $4.04 \pm 0.23$ & -0.52 & 0.607 \\
\hline & Condensation & 14 & $3.99 \pm 0.22$ & $4.01 \pm 0.12$ & -0.40 & 0.690 \\
\hline & Alternating & 14 & $4.02 \pm 0.16$ & $4.02 \pm 0.17$ & -0.06 & 0.947 \\
\hline
\end{tabular}

Mean ABR peak III latencies ( \pm standard deviation) before and after genuine or sham $10 \mathrm{~min} 900 \mathrm{MHz}$ RF exposure generated by a commercial MP and results of the statistical analysis.

three stimulus condition. We found no significant effects of genuine/sham EMF exposure in any of the stimulus conditions.

Figure 1 demonstrates the grand average ABR waveforms recorded in the genuine and sham EMF exposure conditions by rarefaction, condensation and alternating stimuli.

\section{Discussion}

In this study, we found no significant effects of $10 \mathrm{~min}$ genuine MP EMF exposure on the latencies of wave I, III and $\mathrm{V}$ of the auditory brainstem response for rarefaction, condensation or alternating stimuli. Our current results are indirectly confirm the results of earlier investigations demonstrating that 10 minutes of GSM MP exposure does not induce measurable changes in cochlear function [5] in humans, possibly resulting in no deficiencies in the functioning of the central auditory pathways.

The present results reporting no adverse effects are also in line with previous negative results obtained by a similar technique [3]. The authors there studied ABR, and the ABR recovery function as well as middle latency response before and after using a MP for 30 minutes at $0.8 \mathrm{~W}$ power in 15 normal hearing volunteers and found none of these measures affected by exposure to EMF. In a different study [17], the authors investigated the effects of 20 minutes EMF generated by MP on the ABR before, during and after the exposure in 45 young, healthy volunteers. Consistently with our current results, they observed no changes in the latency of waves I, III and V during and after exposure to EMF compared to the initial ABR response.

Contrary, these results are inconsistent with those of a previous pilot study from our laboratory [2], where a single 15 minute MP EMF exposure at maximal $2 \mathrm{~W}$ output power was found to cause a significant delay of $0.207 \mathrm{~ms}$ in the latency of wave $\mathrm{V}$ of the ABR evoked by $80 \mathrm{~dB}$ alternating polarity clicks at $27 \mathrm{~Hz}$ stimulus rate. The authors there suggested that the observed delay might have been caused by the altered functioning of the exposed cochlea due to the absorbed RF energy. However, in the present study, we failed to replicate these previous positive findings. However, in the current study, we administered 10 min of irradiation at $2 \mathrm{~W}$ output power, whereas in the previous study [2] the irradiation lasted for 15 minutes at $2 \mathrm{~W}$ output power. This means that the energy absorbed in the head of the subjects in the present study was about $2 / 3$ of that in the previous study. The shorter irradiation may also account for the present negative findings. In addition, in the previous study, the total number of participants was 10 while in the current study 15 subjects were included in each group, making the statistical power of the current data higher. Taken together, as findings of other studies [3,17] also showed no adverse effects of EMF exposure, we conclusively claim that a single 10 minute GSM MP exposure does not induce significant changes in human ABR. Nevertheless, due to the different exposure conditions of the studies which yielded negative results $[[3,17]]$, the previous positive results [2] must still be taken with caution and indicate the need for additional

Table 3: Mean ABR peak $V$ latencies before and after genuine or sham RF exposure

\begin{tabular}{|c|c|c|c|c|c|c|}
\hline Condition & Stimulus type & $\mathbf{n}$ & Before RF (ms) & After RF (ms) & $\mathbf{t}$ & $\mathbf{p}$ \\
\hline \multirow[t]{3}{*}{ Genuine EMF } & Rarefaction & 13 & $5.89 \pm 0.16$ & $5.93 \pm 0.13$ & -1.67 & 0.120 \\
\hline & Condensation & 13 & $5.81 \pm 0.09$ & $5.83 \pm 0.15$ & -0.51 & 0.613 \\
\hline & Alternating & 13 & $5.85 \pm 0.11$ & $5.88 \pm 0.14$ & -0.78 & 0.450 \\
\hline \multirow[t]{3}{*}{ Sham EMF } & Rarefaction & 15 & $5.75 \pm 0.17$ & $5.76 \pm 0.18$ & -0.48 & 0.634 \\
\hline & Condensation & 14 & $5.76 \pm 0.19$ & $5.72 \pm 0.15$ & 0.41 & 0.684 \\
\hline & Alternating & 14 & $5.81 \pm 0.18$ & $5.82 \pm 0.15$ & -0.40 & 0.696 \\
\hline
\end{tabular}

Mean $\mathrm{ABR}$ peak $\mathrm{V}$ latencies ( \pm standard deviation) before and after genuine or sham $10 \mathrm{~min} 900 \mathrm{MHz} \mathrm{RF}$ exposure generated by a commercial MP and results of the statistical analysis. 
GENUINE EMF
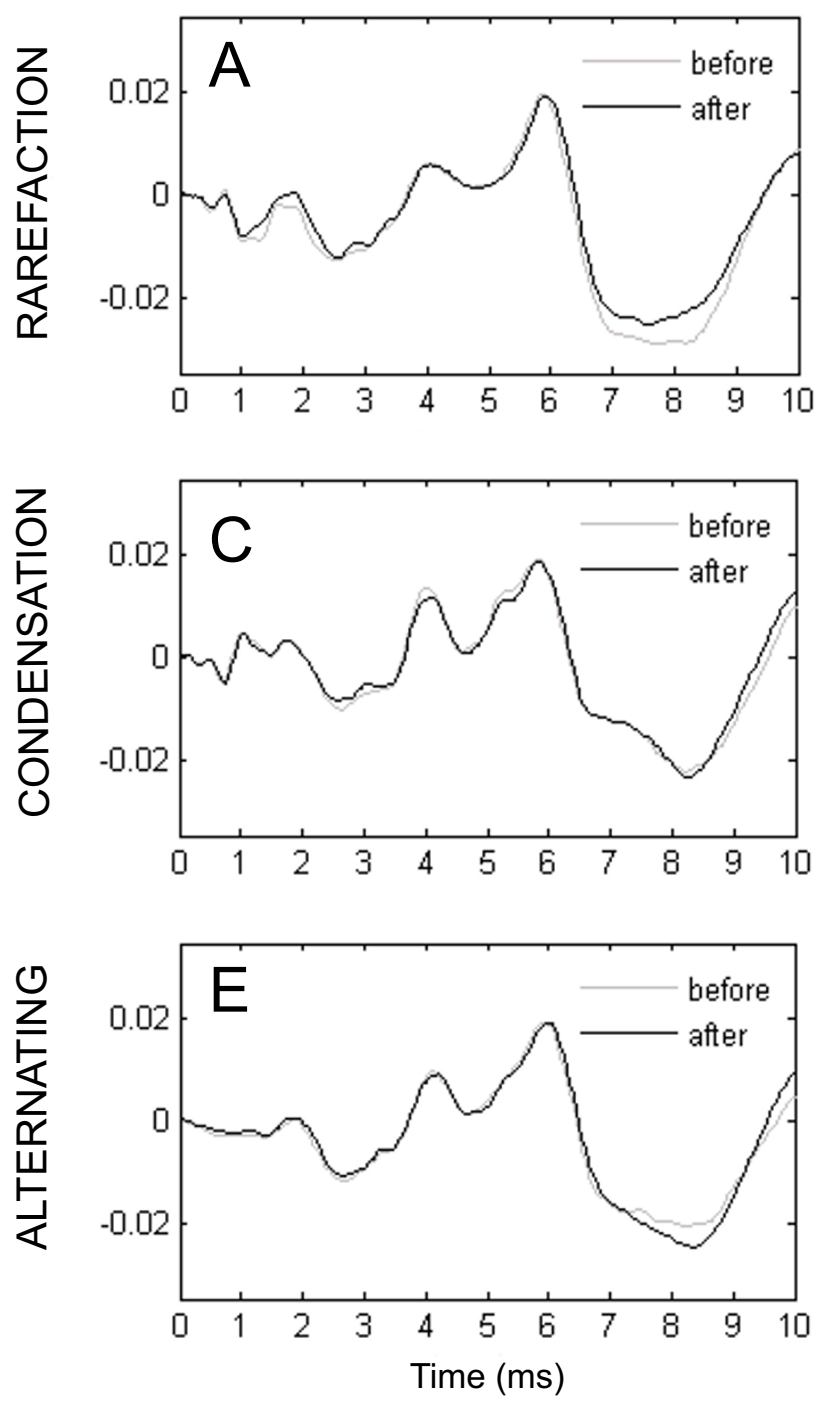

SHAM EMF
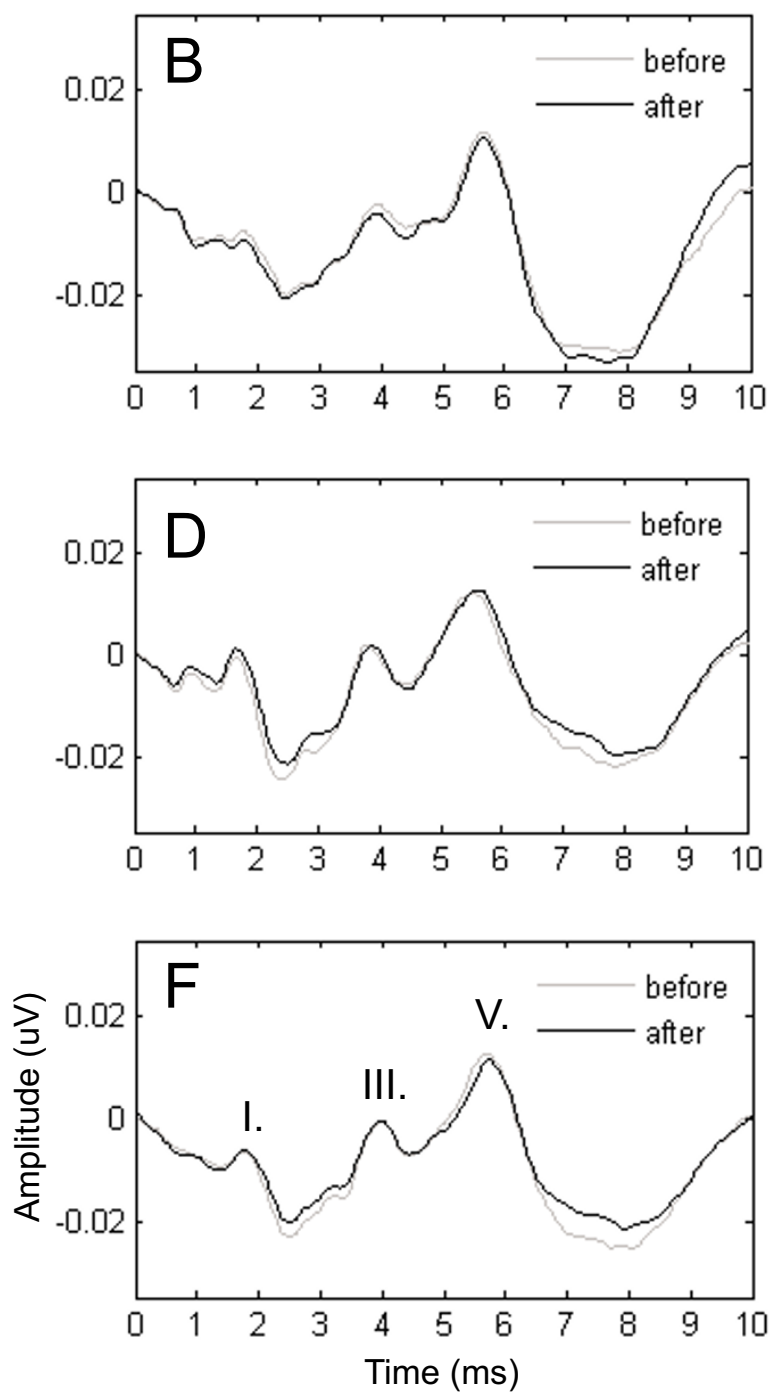

Figure I

Grand average $A B R$ waveforms recorded before and after genuine or sham RF exposure. Grand average $A B R$ waveforms recorded before and after genuine or sham RF exposure for rarefaction (A), condensation $(C)$ and alternating $(E)$ stimuli. Panels on the left show ABR waveforms recorded before and after genuine EMF exposure, while panels to the right depicts $A B R$ waveforms recorded before and after sham EMF exposure for rarefaction (B), condensation (D) and alternating (F) stimuli.

control experiments. In future studies concerning cochlear microphony it would also be important to investigate any possible effects of EMF exposure on the functions of the cochlea.

\section{Conclusion}

Our present results demonstrated that a single 10 minuteexposure to EMF from commercial GSM MPs does not induce measurable effects in ABR peak latencies and may not cause hearing loss, as it was also proposed elsewhere. However, as the potential adverse effects of longer or chronic EMF exposures have not yet been systematically tested, additional experiments are needed to reveal any possible adverse effects of EMFs on the hearing system of humans. In addition, as new generations of mobile sources of EMFs (e.g., 3 G system phones) are being rap- 
idly introduced, it is of utmost importance to establish whether or not these new generations of MPs have potential adverse effects on brain functions in humans.

\section{Competing interests}

The author(s) declare that they have no competing interests.

\section{Authors' contributions}

LK and GT participated in study design. GS and LK have collected the data. FM, GK and GT performed SAR measurements. GS and IH analyzed the data. GS, IH and GT wrote the paper. All authors have read and approved the final manuscript.

\section{Acknowledgements}

This study was supported by the European Commission's GUARD Project (QLK4-CT-200I-00I50). We thank the support from the Graduate School of Biology at the University of Pécs and the Marie Curie Fellowship (to GS). $\mathrm{IH}$ was in receipt of the Bolyai Research Fellowship of the Hungarian Academy of Sciences. We also wish to thank the two reviewers for their valuable comments on the manuscript.

\section{References}

I. Gandhi OP: Electromagnetic fields: human safety issues. Annu Rev Biomed Eng 2002, 4:2। I-234.

2. Kellényi L, Thuróczy G, Faludi B, Lénárd L: Effects of mobile GSM radiotelephone exposure on the auditory brainstem response (ABR). Neurobiology 1999, 7:79-8I.

3. Arai N, Enomoto H, Okabe S, Yuasa K, Kamimura Y, Ugawa Y: Thirty minutes mobile phone use has no short-term adverse effects on central auditory pathways. Clin Neurophysiol 2003, I 14:1390-1394.

4. Parazzini M, Bell S, Thuroczy G, Molnar F, Tognola G, Lutman ME, Ravazzani P: Influence on the mechanisms of generation of distortion product otoacoustic emissions of mobile phone exposure. Hear Res 2005, 208(I-2):68-78.

5. Uloziene I, Uloza V, Gradauskiene E, Saferis V: Assessment of potential effects of the electromagnetic fields of mobile phones on hearing. BMC Public Health 2005, 5:39.

6. Hamblin DL, Wood AW, Croft RJ, Stough C: Examining the effects of electromagnetic fields emitted by GSM mobile phones on human event-related potentials and performance during an auditory task. Clin Neurophysiol 2004, I I5: I7I-178.

7. Hamblin DL, Croft RJ, Wood AW, Stough C, Spong J: The Sensitivity of Human Event-Related Potentials and Reaction Time to Mobile Phone Emitted Electromagnetic Fields. Bioelectromagnetics 2006, 27:265-273.

8. Yuasa K, Arai N, Okabe S, Tarusawa Y, Nojima T, Hanajima R, Terao $Y$, Ugawa $Y$ : Effects of thirty minutes mobile phone use on the human sensory cortex. Clin Neurophysiol 2006, I I 7:900-905.

9. Preece AW, Iwi G, Davies-Smith A, Wesnes K, Butler S, Lim E, Varey A: Effect of a 915-MHz simulated mobile phone signal on cognitive function in man. Int J Radiat Biol 1999, 75:447-456.

10. Koivisto M, Revonsuo A, Krause C, Haarala C, Sillanmäki L, Laine M, Hämäläinen $\mathrm{H}$ : Effects of $\mathbf{9 0 2} \mathbf{~ M H z}$ electromagnetic field emitted by cellular telephones on response times in humans. NeuroReport 2000, I I:4I3-4I5.

II. Koivisto M, Krause CM, Revonsuo A, Laine M, Hämäläinen H: The effects of electromagnetic field emitted by GSM phones on working memory. NeuroReport 2000, I I:164I-I643.

12. Preece AW, Goodfellow S, Wright MG, Butler SR, Dunn EJ, Johnson Y, Manktelow TC, Wesnes K: Effect of 902 MHz mobile phone transmission on cognitive function in children. Bioelectromagnetics 2005:SI38-143.

13. Haarala C, Ek M, Bjornberg L, Laine M, Revonsuo A, Koivisto M, Hämäläinen $\mathrm{H}: \mathbf{9 0 2} \mathbf{~ M H z}$ mobile phone does not affect short term memory in humans. Bioelectromagnetics 2004, 25:452-456.
14. Krause CM, Sillanmäki L, Koivisto M, Häggqvist A, Saarela C, Revonsuo $A$, Laine $M$, Hämäläinen $H$ : Effects of electromagnetic field emitted by cellular phones on the EEG during a memory task. NeuroReport 2000, I I:76I-764.

15. Papageorgiou CC, Nanou ED, Tsiafakis VG, Kapareliotis E, Kontoangelos KA, Capsalis CN, Rabavilas AD, Soldatos CR: Acute mobile phone effects on pre-attentive operation. Neurosci Lett 2006, 397:99-103.

16. GUARD projects: "Potential Adverse Effects of GSM Cellular Phones on Hearing". [http://www.guard.polimi.it].

17. Bak M, Sliwinska-Kowalska M, Zmyslony M, Dudarewicz A: No effects of acute exposure to the electromagnetic field emitted by mobile phones on brainstem auditory potentials in young volunteers. Int J Occup Med Environ Health 2003, 16:20I-208.

\section{Pre-publication history}

The pre-publication history for this paper can be accessed here:

http://www.biomedcentral.com/1471-2458/7/325/pre pub
Publish with BioMed Central and every scientist can read your work free of charge

"BioMed Central will be the most significant development for disseminating the results of biomedical research in our lifetime. " Sir Paul Nurse, Cancer Research UK

Your research papers will be:

- available free of charge to the entire biomedical community

- peer reviewed and published immediately upon acceptance

- cited in PubMed and archived on PubMed Central

- yours - you keep the copyright

Submit your manuscript here:

http://www.biomedcentral.com/info/publishing_adv.asp
BioMedcentral 